Review paper

\title{
Photon-counting CT review
}

\author{
Thomas Flohr ${ }^{\text {a, }}$, Martin Petersilka ${ }^{\text {a }}$, Andre Henning ${ }^{\text {a }}$, Stefan Ulzheimer ${ }^{a}$, Jiri Ferda ${ }^{\mathrm{b}}$, \\ Bernhard Schmidt ${ }^{\mathrm{a}}$ \\ a Siemens Healthcare GmbH, Computed Tomography, 91301 Forchheim, Germany \\ ${ }^{\mathrm{b}}$ Charles University Prague, Medical Faculty Pilsen, 30460 Pilsen, Czech Republic
}

\section{A R T I C L E I N F O}

\section{Keywords:}

Computed tomography CT

Photon-counting detector

Photon-counting CT

Spectral CT

\begin{abstract}
A B S T R A C T
Photon-counting detectors are a promising new technology for computed tomography (CT) systems. They provide energy-resolved CT data at very high spatial resolution without electronic noise and with improved tissue contrasts. This review article gives an overview of the principles of photon-counting detector CT, of potential clinical benefits and limitations, and of the experience gained so far in pre-clinical installations.
\end{abstract}

\section{Introduction}

Computed tomography (CT) is the backbone of radiological diagnosis. The application spectrum of CT has been continuously expanded by technological advances, among them the introduction of spiral CT [1], the rapid progress of multi-detector row CT [2,3], and new system concepts such as wide detector CT [4] or dual source CT [5] with their specific clinical benefits.

Today, CT is a mature modality in its saturation phase. Yet, there are still some limitations for current CT technology.

Limited spatial resolution hampers CT angiographic (CTA) examinations of small vessels such as the coronary arteries. Calcified plaques in the vessels appear much larger in the CT-images than they are ("Cablooming"), and coronary narrowing is frequently overestimated. Coronary calcifications with an Agatston score greater than 1000 are the most relevant independent predictor of uninterpretable coronary CTAs [6]. Other CTAs, such as renal CTAs for surgery planning or peripheral run-offs, and lung examinations, e. g. for interstitial lung disease, will benefit from improved spatial resolution as well. Recently, an ultrahigh-resolution CT system was introduced (Canon Precision). The system has been evaluated in initial studies [7], routine clinical experience, however, is still limited.

Excessive increase of image noise, noise streaks and drift of CTnumbers in CT scans of obese patients or at ultra-low radiation dose are another drawback of current CT. They are caused by the electronic noise of the measurement system which dominates at low x-ray flux. Detectors with integrated detector electronics show reduced electronic noise [8] and may enable further radiation dose reduction.
Dual-energy CT has gained momentum as a technique to enhance the clinical value of CT by providing functional information in addition to morphology. Dual-energy CT exploits the material-specific difference in $\mathrm{x}$-ray attenuation at different $\mathrm{x}$-ray energies and has resulted in a variety of clinical applications:

- Material differentiation, e. g. for the characterization of kidney stones or differential diagnosis of gout

- Improved visualization, e. g. by means of contrast enhancement of lesions and other structures with the help of virtual monoenergetic images (VMIs)

- Quantitation, e. g. by computation of iodine maps, with the local iodine uptake as a surrogate parameter for local perfusion in patients with pulmonary embolism, or for differential diagnosis of abdominal lesions.

Reviews on clinical applications of dual-energy CT may be found in $([9,10,11,12,13,14,15])$. Dual-energy CT data can currently be acquired with dual source CT systems $([5,16])$, CT systems with fast $\mathrm{kV}$ switching [17] or dual-layer detector CT systems [18]. However, each of these solutions has inherent limitations.

Photon-counting detectors are a new technology with the potential to overcome the above-mentioned limitations of current CT detectors, by providing CT data at very high spatial resolution, without electronic noise and with inherent spectral information. Photon-counting detectors and their potential benefits have already been evaluated in experimental CT benchtop systems more than 10 years ago (e. g. [19]). The performance of the detectors used in these early systems, however, was not

\footnotetext{
* Corresponding author.

E-mail address: thomas.flohr@siemens-healthineers.com (T. Flohr).
} 


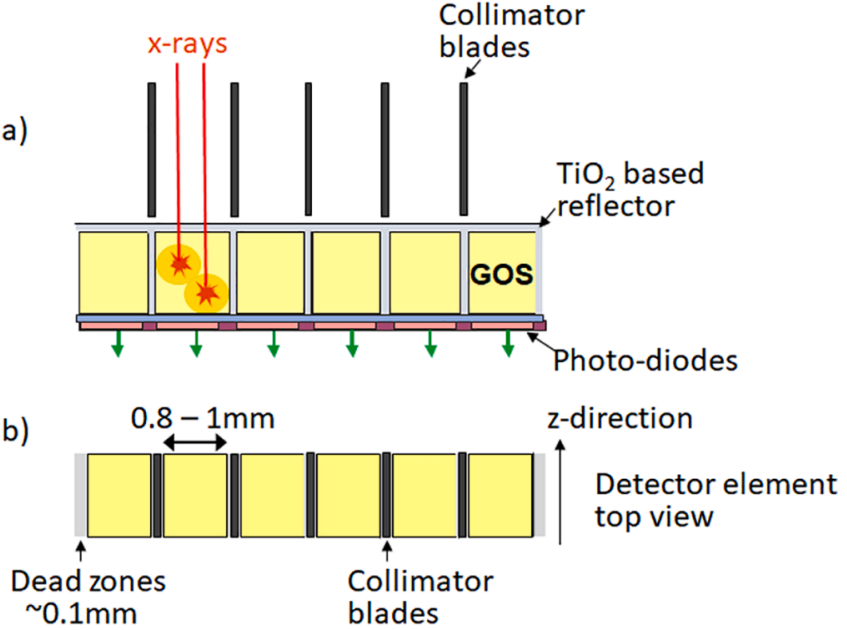

Fig. 1. Schematic drawing of an energy-integrating scintillator detector. a) side view, b) top view. The z-direction is the patient's longitudinal direction. Detector cells made of a scintillator such as GOS absorb the x-rays (red arrows) and convert their energy into visible light (orange circles). This light is detected by photodiodes and converted into an electrical current. The individual detector cells are separated by optically in-transparent layers (e. g. based on $\mathrm{TiO}_{2}$ ) to prevent optical crosstalk ("dead zones"). Collimator blades above the separation layers suppress scattered radiation. (For interpretation of the references to color in this figure legend, the reader is referred to the web version of this article.)

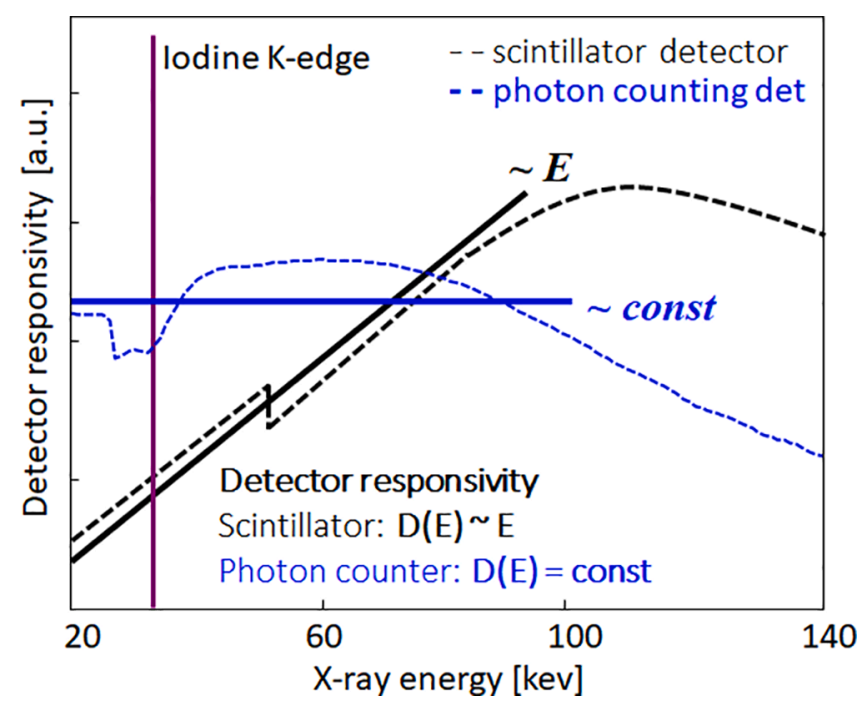

Fig. 2. Detector responsivity $D(E)$ as a function of the $x$-ray energy $E$ for a GOS scintillator detector (dotted black line) and a CdTe photon-counting detector (dotted blue line). The iodine $\mathrm{K}$-edge at $33 \mathrm{keV}$ is indicated by a vertical line. Low-energy $\mathrm{x}$-rays closely above the K-edge of iodine contribute less to the detector signal of a scintillator detector than of a photon-counting detector. At the same $\mathrm{x}$-ray tube voltage the iodine contrast in the image is therefore lower for a scintillator detector than for a photon-counting detector. (For interpretation of the references to color in this figure legend, the reader is referred to the web version of this article.)

adequate for clinical CT imaging, mainly because they did not tolerate the high x-ray flux rates of medical CT. Significant recent progress in detector material synthesis and detector electronics design has meanwhile enabled the installation of pre-clinical whole-body photoncounting CT prototypes and pre-clinical testing in human subjects. Despite all technical advances, significant development efforts are still needed before the detectors can be broadly released in commercial CT systems.

This review article gives an overview of the basic principles of photon-counting detector CT, and of the clinical experience gained so far in pre-clinical installations. Other reviews of photon-counting detector CT may be found in $([20,21,22,23])$.

\section{Principles of photon-counting CT}

\subsection{Properties of current solid-state scintillation detectors}

To understand the properties of photon-counting CT detectors and their clinical impact it is helpful to briefly recapitulate the properties of solid-state scintillation detectors which are used in all current medical CT scanners. Solid-state scintillation detectors consist of individual detector cells with a side length of $0.8-1 \mathrm{~mm}$, made of a scintillator (e. g. Gadolinium-oxide or Gadolinium-oxysulfide GOS) with a photodiode attached to its backside, see Fig. 1 . The absorbed x-rays produce visible light in the scintillator which is detected by the photodiode and converted into an electrical current. Both the intensity of the scintillation light and the amplitude of the induced current pulse are proportional to the energy $E$ of the absorbed x-ray photon. All current pulses registered during the measurement time of one reading (projection) are integrated. This results in a detector signal $S$

$S=\int_{0}^{E m a x} D(E) N(E) d E \approx \int_{0}^{E m a x} E N(E) d E$

where $D(E)$ is the detector responsivity, $N(E)$ is the absorbed x-ray flux during one reading, and $E_{\max }$ is the maximum energy of the x-ray beam, see Fig. 2.

X-ray photons with lower energy $E$, which carry most of the lowcontrast-information, contribute less to the integrated detector signal than x-ray photons with higher energy. This energy-weighting reduces the contrast-to-noise ratio (CNR) in the CT images, in particular in CT scans with iodinated contrast agent, because the $\mathrm{x}$-ray absorption of iodine is highest at lower energies closely above its K-edge at $33 \mathrm{keV}$.

The low-level analogue electric signal of the photodiodes is distorted by electronic noise which becomes larger than the quantum noise (Poisson noise) of the x-ray photons at low x-ray flux and causes a disproportional increase of image noise and instability of low CTnumbers (e. g. in the lungs) if the flux is further reduced. This strong noise increase and the drift of CT-numbers set a limit to potential further radiation dose reduction in medical CT.

The individual detector cells are separated by optically intransparent layers to prevent optical crosstalk. They have a minimum width of about $0.1 \mathrm{~mm}$ and reduce the geometric dose efficiency of the detector: x-ray photons absorbed in the separation layers do not contribute to the measured signal even though they have passed through the patient - from a radiation dose perspective they are wasted dose. Current medical CT detectors have detector cells with an active size of about $0.8 \times 0.8 \mathrm{~mm}^{2}$ to $1 \times 1 \mathrm{~mm}^{2}$ [22]. Assuming additional dead zones of $0.2 \mathrm{~mm}$ in the in-plane direction and $0.1 \mathrm{~mm}$ in the $z$-axis direction, the total area of a detector element is $1.0 \times 0.9 \mathrm{~mm}^{2}$ to $1.2 \times 1.1 \mathrm{~mm}^{2}$. The geometric dose efficiency is therefore about $70-80 \%$. Significantly reducing the size of the scintillators to increase spatial resolution while keeping the width of the separation layers constant will further reduce the geometric efficiency - therefore, it is problematic to increase the spatial resolution of solid-state scintillation detectors beyond today's performance levels, see also [69].

\subsection{Properties of photon-counting detectors}

Photon-counting detectors are made of semiconductors such as cadmium-telluride (CdTe), cadmium-zinc-telluride (CZT) or silicon (Si). Depending on the material, the detector consists of a 1.4-30 mm thick semiconductor layer [22]. Thin layers are sufficient for CdTe- and CZT- 


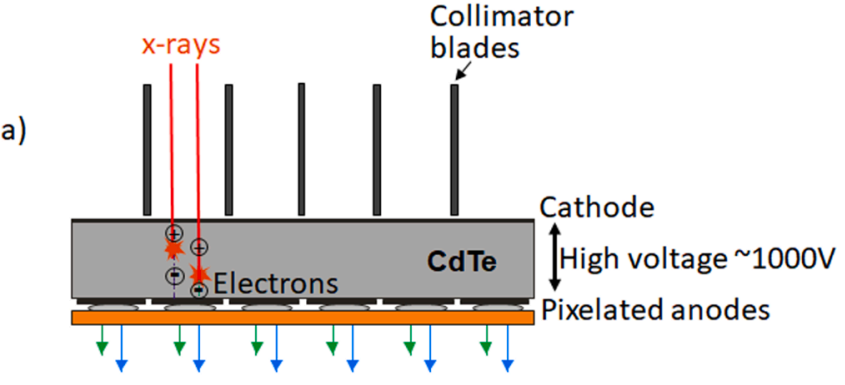

b)

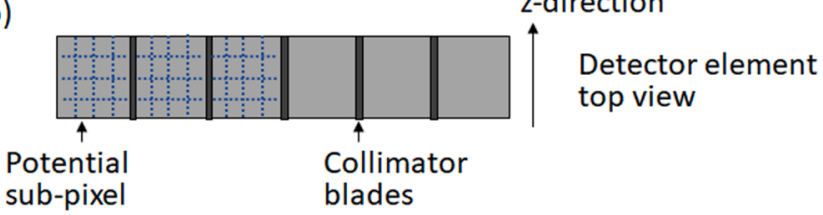

structu

Fig. 3. Schematic drawing of a direct converting photon-counting detector. a) side view, b) top view. The z-direction is the patient's longitudinal direction. The x-rays (red arrows) absorbed in a semiconductor such as CdTe or CZT produce electron-hole pairs that are separated in a strong electric field between cathode and pixelated anodes. The detector pixels are formed by the pixelated anodes and the electric field, there are no separation layers between them. Collimator blades are needed to suppress scattered radiation. Different from scintillator detectors each "macro pixel" between two collimator blades can be divided into smaller sub-pixels because of the absence of "dead zones", as indicated for the three left detector cells. Of course, the pixelated anodes must then be correspondingly structured, which is not shown here in order not to overload the drawing. (For interpretation of the references to color in this figure legend, the reader is referred to the web version of this article.)

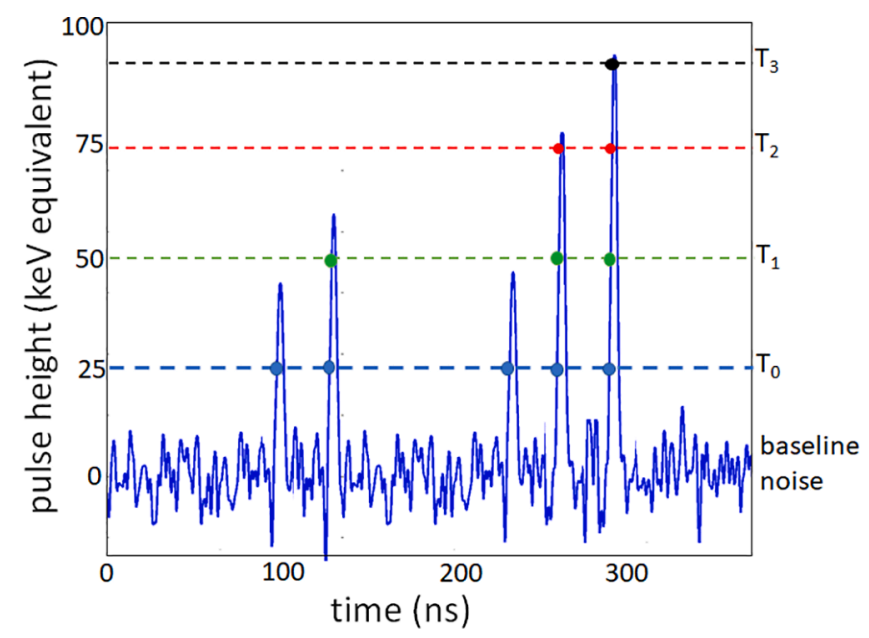

Fig. 4. The electrons produced by absorbed x-rays in a photon-counting detector induce signal pulses at the anodes with a pulse height proportional to the $\mathrm{x}$-ray energy. The pulses are counted as soon as they exceed a threshold $\mathrm{T}_{0}$ (dashed blue line, "counting" is indicated by a blue dot). $\mathrm{T}_{0}$ has a typical energy of $25 \mathrm{keV}$, well above the low-amplitude baseline noise. Three additional thresholds at higher energies $\left(\mathrm{T}_{1}\right.$ at $50 \mathrm{keV}, \mathrm{T}_{2}$ at $75 \mathrm{keV}, \mathrm{T}_{3}$ at $90 \mathrm{keV}$ ) are also indicated - simultaneous read-out of the counts at various energy thresholds (in this example 4) provides spectrally resolved detector signals. (For interpretation of the references to color in this figure legend, the reader is referred to the web version of this article.)

based CT detectors because of their high atomic number. Si requires thick layers because of its low atomic number and low absorption efficiency in the x-ray energy range relevant to medical CT [64]. In this review we focus on CdTe- and CZT-based photon counting detectors.

High voltage (800-1000 V) is applied between the cathode on top and pixelated anode electrodes at the bottom of the semiconductor layer, see Fig. 3. The absorbed $\mathrm{x}$-rays produce electron-hole pairs which are separated in the strong electric field. The electrons drift to the anodes and induce short current pulses $\left(10^{-9} \mathrm{~s}\right)^{1}$. A pulse-shaping circuit transforms them to voltage pulses with a full width at half maximum (FWHM) of 10-15 ns; the pulse-height of the voltage pulses is proportional to the energy $E$ of the absorbed x-ray photons. As soon as these pulses exceed a threshold they are counted, see Fig. 4.

Photon-counting detectors have several advantages compared to solid-state scintillation detectors. The individual detector cells are defined by the strong electric field between common cathode and pixelated anodes (Fig. 3), there are no additional separation layers. The geometrical dose efficiency of a photon-counting detector is only reduced by the unavoidable anti-scatter collimator blades or grids. Different from scintillator detectors each "macro pixel" confined by collimator blades can be divided into smaller sub-pixels which are readout separately to increase spatial resolution (see Fig. 3b).

All current pulses produced by absorbed x-rays are counted during the measurement time of one reading (projection) as soon as they exceed a threshold energy $\mathrm{T}_{0}$. In a photon-counting detector for medical CT, $\mathrm{T}_{0}$ is about $20-25 \mathrm{keV}$. Low-amplitude baseline noise is well below this level and does not trigger counts - even at low x-ray flux only the statistical Poisson noise of the $\mathrm{x}$-ray quanta is present in the signal. CT scans at very low radiation dose or CT scans of obese patients show therefore less image noise, less streak artifacts and more stable CT-numbers than the corresponding scans with a scintillation detector, and radiation dose reduction beyond today's limits seems possible.

The detector responsivity $\mathrm{D}(\mathrm{E})$ in the $\mathrm{x}$-ray energy range from $30 \mathrm{keV}$ to $100 \mathrm{keV}$ is approximately constant (see Fig. 2) -all x-ray photons contribute equally to the measurement signal regardless of their energy $E$, as soon as $E$ exceeds $\mathrm{T}_{0}$. This results in a detector signal $S$

$S=\int_{T 0}^{E \max } D(E) N(E) d E \approx \int_{T 0}^{E \max } \operatorname{const} N(E) d E$

There is no down-weighting of lower energy x-ray photons as in solid-state scintillation detectors. Photon-counting detectors can therefore provide CT images with potentially improved CNR, in particular in CT scans with iodinated contrast agent.

In a more advanced readout mode, several counters operating at different threshold energies are introduced for energy discrimination, see Fig. 4. Physically, the different thresholds are realized by different voltages which are fed into pulse-height comparator circuits. In the example of Figs. 4, 4 different energy thresholds $T_{0}, T_{1}, T_{2}$ and $T_{3}$ are realized. During the time of one projection, counter 1 counts all $\mathrm{x}$-ray pulses with an energy exceeding $\mathrm{T}_{0}$, while counter 2 simultaneously counts all $\mathrm{x}$-ray pulses with an energy exceeding $\mathrm{T}_{1}$, and so on. The photon-counting detector simultaneously provides 4 signals $S_{0}, S_{1}, S_{2}$ and $S_{3}$ with different lower energy thresholds $\mathrm{T}_{0}, \mathrm{~T}_{1}, \mathrm{~T}_{2}$ and $\mathrm{T}_{3}$.

$S_{i}=\int_{T i}^{E m a x} D(E) N(E) d E, i=0,1,2,3$

CT images reconstructed from these raw data are shown in Fig. 5. By subtracting the detector signals with adjacent lower energy thresholds, "energy bin" data can be produced. Energy bin $b_{0}=S_{1}-S_{0}$ as an example contains all $\mathrm{x}$-ray photons detected in the energy range between $T_{1}$ and $T_{0}$.

The simultaneous read-out of CT data in different energy bins opens the potential of spectrally resolved measurements and material differentiation in any CT scan. Today's established dual-energy applications mainly based on decomposition into two base materials such as iodine and water, or iodine and calcium - are routinely feasible, and virtual

\footnotetext{
1 The drift of the holes is much slower. Therefore, they can cause polarization at high count rates, significantly impacting the detector response.
} 

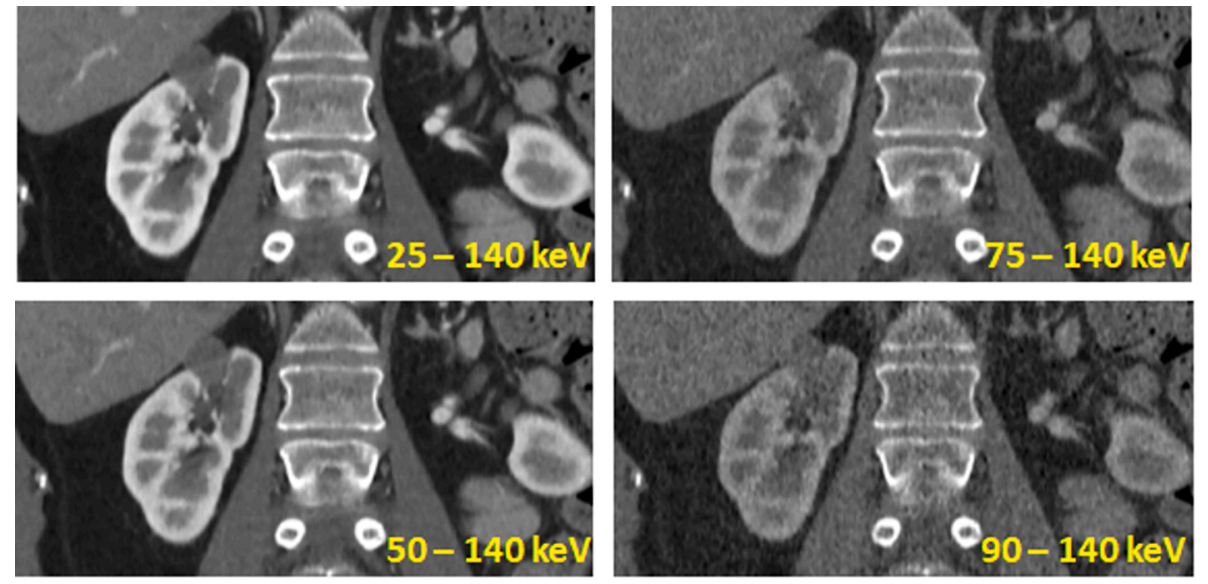

Fig. 5. Contrast-enhanced kidney scan acquired with a pre-clinical hybrid dual source CT prototype with 4 energy thresholds $(25 \mathrm{keV}, 50 \mathrm{keV}, 75 \mathrm{keV}$ and $90 \mathrm{keV}$, as indicated in Fig. 4), operated at an x-ray tube voltage of $140 \mathrm{kV}$. The higher the energy threshold, the lower is the iodine contrast and the higher is the image noise in the reconstructed images, because fewer low-energy x-ray photons contribute to the image. Courtesy of National Institute of Health NIH, Bethesda, MD, USA.
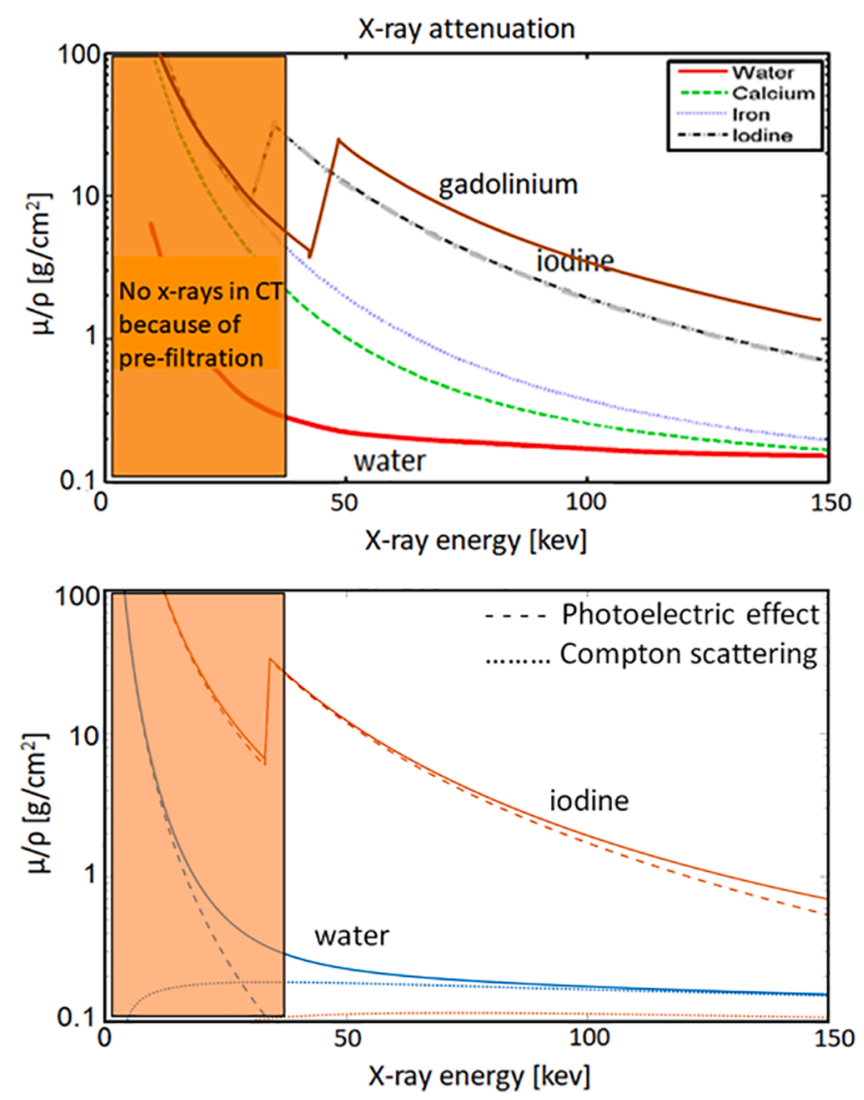

Fig. 6. Top: X-ray attenuation of water, iodine, calcium, iron and gadolinium as a function of the $\mathrm{x}$-ray energy. Differentiation of two base materials, e. g. water and iodine, requires measurements at two different energies. The attenuation of any other material without K-edge (e. g. calcium or iron) can then be described by a linear combination of the two base materials. Three- or more material decomposition is only feasible by adding a third element with K-edge in the relevant energy range, in this example gadolinium with a K-edge at 55 $\mathrm{keV}$. Bottom: Decomposition of the x-ray attenuation of water and iodine into Compton scattering and the photoelectric effect. The attenuation of iodine is dominated by the photoelectric effect because of its higher $Z$.

monoenergetic images, iodine maps or virtual non-calcium images can be computed whenever needed for the diagnosis.

Data acquisition with more than two thresholds enables multi- material decomposition under certain preconditions. Compton scattering and the photoelectric effect are the only two relevant interaction mechanisms for x-ray energies used in medical CT (30 keV - $150 \mathrm{keV})$. Their energy dependence is similar for all elements without K-edge in this energy range - this applies to all elements naturally occurring in the human body. Their x-ray attenuation $\mu(E)$ can be described as

$$
\mu(E)=\rho \cdot\left(\left(\frac{\mu}{\rho}\right)_{\text {Photo }}(E, Z)+\left(\frac{\mu}{\rho}\right)_{\text {Compton }}(E, Z)\right) \approx \rho \cdot\left(\alpha \cdot \frac{Z_{\text {eff }}^{k}}{E^{n}}+\beta \cdot f_{K N}(E)\right)
$$

$\rho$ is the density. The first term $\propto \frac{Z_{e f f}^{k}}{E^{n}}$ describes the attenuation by the photoelectric effect. $Z_{\text {eff }}$ is the effective atomic number, $k \sim 3-4, n \sim$ 3-3.5. The second term $f_{K N}(E)$ is the Klein-Nishina description of attenuation by Compton scattering. $f_{\mathrm{KN}}(E)$ depends only weakly on $E$. $\alpha$ and $\beta$ are constants for all energies and $\mathrm{Z}_{\mathrm{eff}}$ 's.

Differentiation of two base materials, e. g. water and iodine, requires two measurements at different energies. The energy-dependent attenuation of any other material, such as calcium or iron, can then be described by a linear combination of the two base materials, see Fig. 6 . Adding further measurements at other energies will not provide relevant new information in this case. The situation changes if a material with $\mathrm{K}$ edge in the relevant energy range, such as gadolinium, is added to the two base materials, see Fig. 6. For a K-edge material, the energy dependence of x-ray attenuation is different, and CT measurements at three or more energies can be used for three-material decomposition (two base materials plus the K-edge material):

$\mu(E)=\rho \cdot\left(\alpha \frac{Z_{e f f}^{k}}{E^{n}}+\beta f_{K N}(E)+a_{K-e d g e} f_{K-e d g e}(E)\right)$

$f_{K \text {-edge }}(E)$ describes the energy-dependent attenuation by the K-edge element.

Unfortunately, three- or more-material decomposition with CT data in three or more energy bins will be limited to clinical scenarios in which K-edge elements have been introduced into the human body, e. g. to separate two contrast agents (e. g. iodine and gadolinium, or iodine and bismuth) or other heavy elements (e. g. tungsten, or gold nanoparticles).

In addition to potential material decomposition, the CNR of the images can be further improved by optimized weighting of the different energy bins. Instead of just adding the bin data for the reconstruction of an image, higher weights can be assigned to the low-energy bin data, in particular in CT-scans using iodinated contrast agent.

\subsection{Challenges for photon-counting detectors}

Compared to established dual-energy acquisition techniques, 


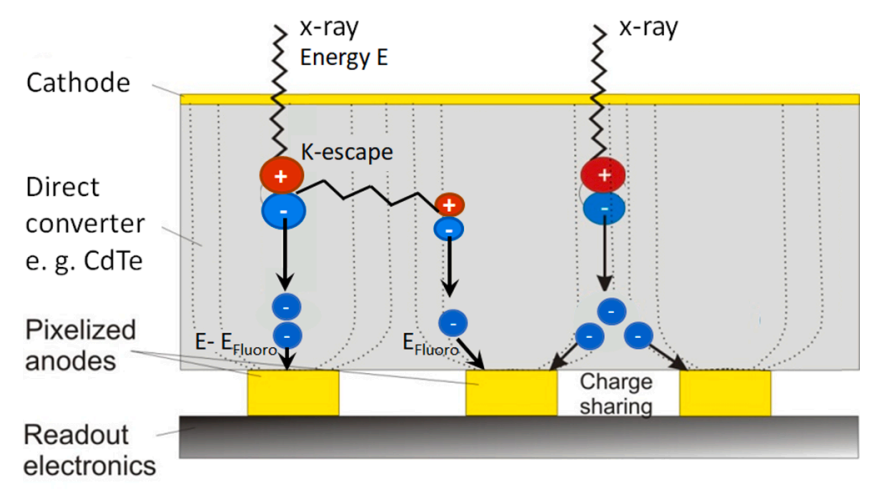

Fig. 7. Schematic illustration of charge sharing at pixel boundaries and energy loss due to K-escape, which lead to double counting of x-ray pulses at wrong energies and reduction of spectral separation. $\mathrm{E}_{\text {Fluoro }}$ is the K-shell fluorescence $\mathrm{x}$-ray energy.

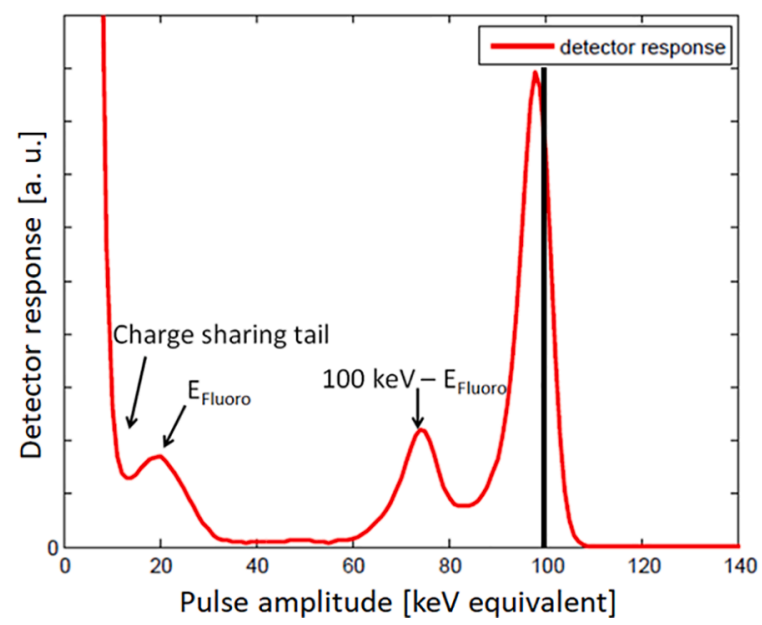

again wrongly counted at lower energies, and spectral separation as well as spatial resolution are reduced. Charge sharing, fluorescence and Kescape are illustrated in Fig. $7 .^{2}$

Fig. 8 shows the spectral response of a CdTe-photon-counting detector $^{3}$. Fig. 8 serves as a qualitative illustration of the influence of charge sharing, fluorescence and K-escape on the detector signal, it is not intended to provide quantitative information about the performance of a particular CdTe/CZT-detector. If the detector is read-out with several energy thresholds, resulting in several energy bins, the low energy bins will contain wrong high-energy information (because highenergy hits result in detector response at lower x-ray energies, see Fig. 8). For a realistic detector model including charge sharing, fluorescence, and K-escape, the spectral separation with two energy bins is probably equivalent to that of a dual-kV scan with optimized prefiltration [24].

Increasing the size of the detector pixels improves spectral separation, because boundary effects such as charge sharing and K-escape contribute less to the total detector signal, see Fig. 9.

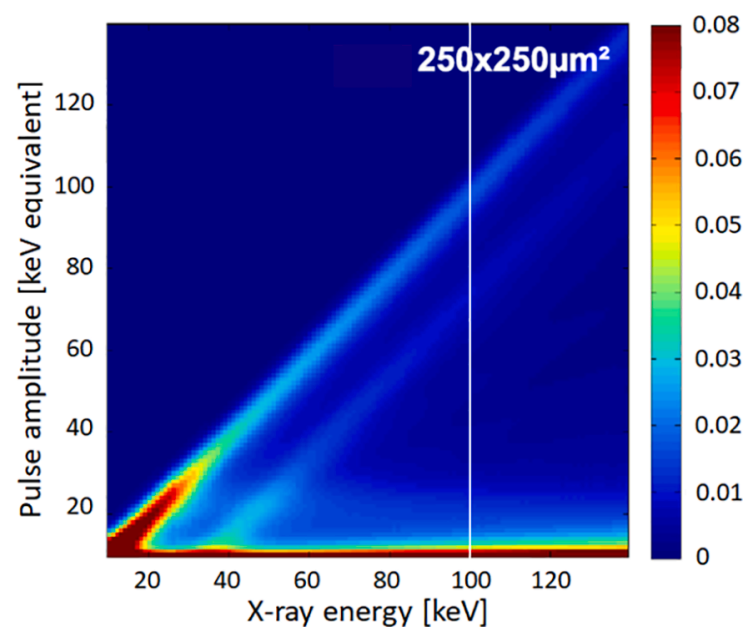

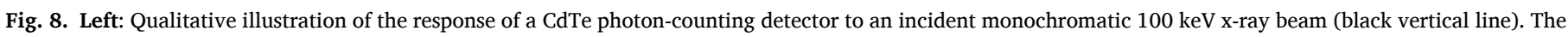

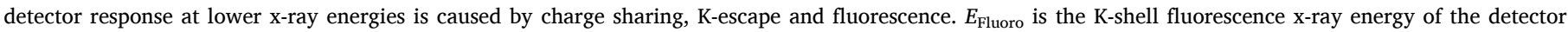

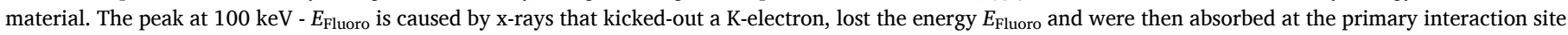

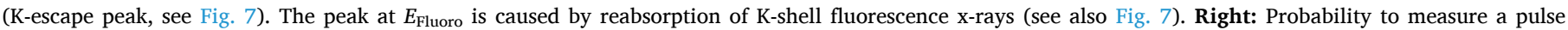

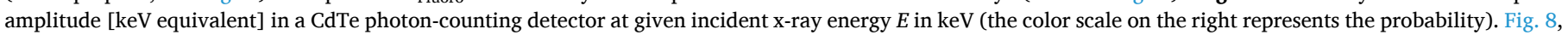
left, can be regarded as a vertical cut through this diagram at $100 \mathrm{keV}$, see the white line (modified from [70]).

photon-counting detectors are often assumed to provide better energy separation and less spectral overlap. However, unavoidable physical effects reduce the energy separation of CdTe- or CZT-based photoncounting detectors. The current pulses produced by $\mathrm{x}$-rays absorbed close to pixel borders are split between adjacent detectors cells ("charge sharing"). This leads to erroneous counting of a high-energy x-ray photon as several lower-energy hits. Cd and Te have K-edges at 26.7 and $31.8 \mathrm{keV}$, respectively. Incident $\mathrm{x}$-rays at an energy $E$ may kick-out Kelectrons of the detector material. The empty K-shells are immediately refilled, and characteristic x-rays at the K-shell fluorescence energy $E_{\text {Fluoro }}$ are released which are re-absorbed and counted in the detector cell itself or in neighboring detector cells ("K-escape"). The incident $\mathrm{x}$ rays at the primary interaction site lose the energy $E_{\text {Fluoro }}$ and are counted at an energy $E$ - $E_{\text {Fluoro }}$ (the resulting peak in the detector signal is called "K-escape peak"). In summary, high-energy x-ray photons are
The maximum size of the detector pixels is unfortunately limited by the finite width of the voltage pulses after pulse-shaping (FWHM $\geq 10$ ns). Medical CTs are operated at high $\mathrm{x}$-ray flux rates up to $10^{9}$ counts per $\mathrm{s}$ and $\mathrm{mm}^{2}-$ if the detector pixels are too large, too many $\mathrm{x}$-ray

\footnotetext{
${ }^{2} \mathrm{Si}$-based photon counting detectors do not suffer from problems with $\mathrm{K}$ escape. However, because of the low atomic number of $\mathrm{Si}$, a large number of the incident x-ray photons are not photo-absorbed, but one or more times Comptonscattered, each time depositing a small amount of energy. The Compton interactions degrade both energy separation and spatial resolution [64]

${ }^{3}$ Details of the simulation software are described in $[70,73,74]$. We assumed a $1.6 \mathrm{~mm}$ thick direct converter with pixelized anodes (cell size $250 \times 250$ $\mu \mathrm{m}^{2}$ ). A bias voltage of $1000 \mathrm{~V}$ was applied between cathode and anode pixels. We assumed analogue preamplifiers with Gaussian shaping characteristics, resulting in signal pulses of 25 ns FWHM pulse duration in average. Fluorescence, K-escape, charge diffusion/dispersion, and signal splitting at sub-pixel borders were taken into account. We did not account for pulse pile-up. We assumed a CT-typical X-ray spectrum from a tungsten anode with standard prefiltration of $0.9 \mathrm{~mm}$ titanium and $3.5 \mathrm{~mm}$ aluminum. Comprehensive simulations of the energy response of photon counting detectors including variation of physical parameters such as pixel size and pulse pile-up are available in [71-74].
} 

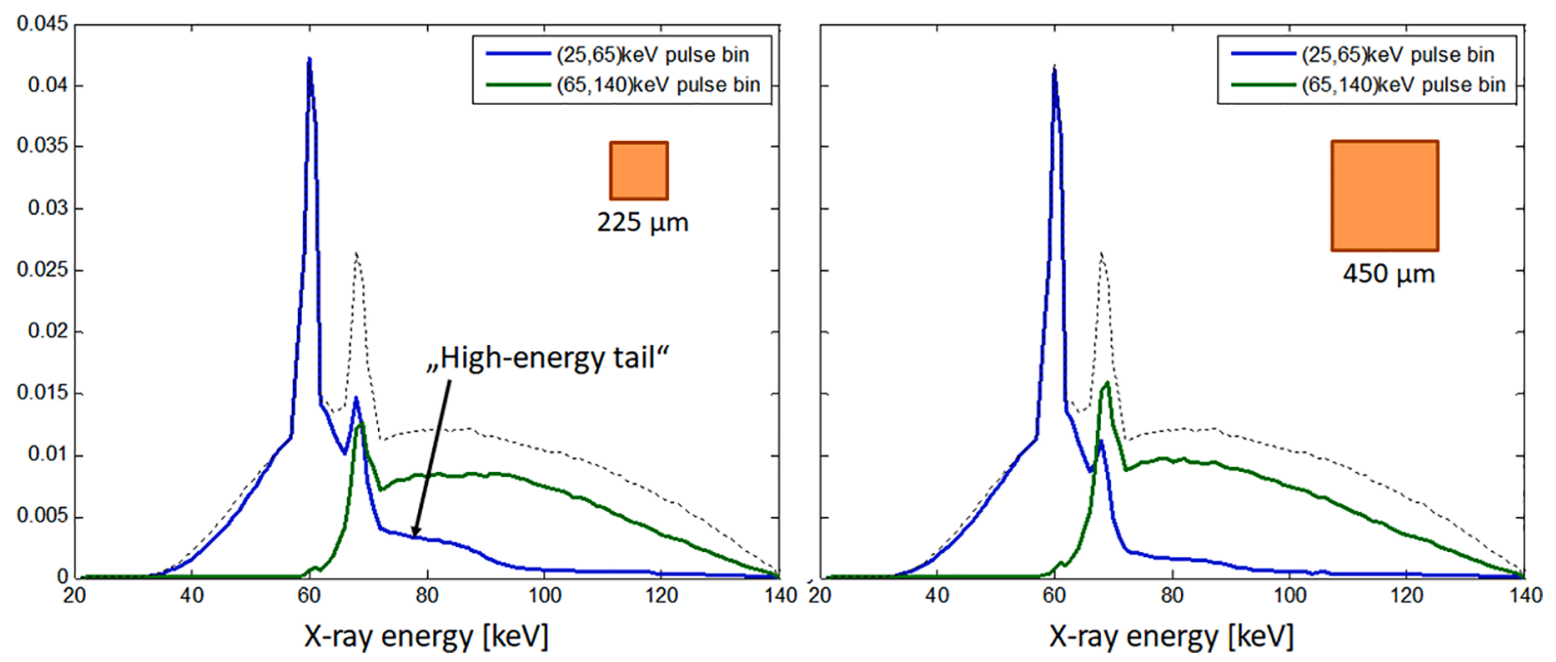

Fig. 9. Computer simulation of the $\mathrm{x}$-ray spectra recorded in the 2 energy bins of a realistic photon-counting detector for an incident $140 \mathrm{kV}$ spectrum (bin $1: 25-65$ $\mathrm{keV}$, blue line; bin $_{2}: 65-140 \mathrm{keV}$, green line). The dotted line is the incident $\mathrm{x}$-ray spectrum. Left: pixel size $0.225 \times 0.225 \mathrm{~mm}^{2}$. Right: pixel size $0.45 \times 0.45 \mathrm{~mm}^{2}$. Increasing the pixel size reduces the characteristic "high-energy tail" of the low energy bin caused by charge sharing and other effects such as K-escape. (For interpretation of the references to color in this figure legend, the reader is referred to the web version of this article.)

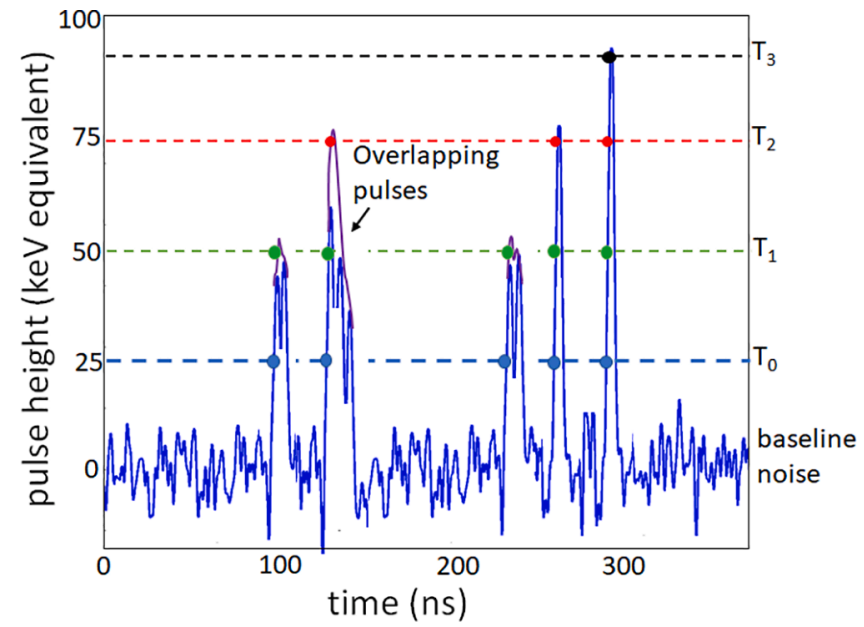

Fig. 10. Schematic illustration of pulse pile-up in a photon-counting detector with 4 energy thresholds (compare to the situation in Fig. 4). Because of the higher x-ray flux rate than in Fig. 4, some of the signal pulses overlap and are not registered separately, but as one hit only (pink lines) at the wrong energy thresholds ("pulse pile-up"). The larger the detector pixels are, the lower are the $\mathrm{x}$-ray flux rates that can be registered without pulse pile-up. (For interpretation of the references to color in this figure legend, the reader is referred to the web version of this article.)

photons hit them too closely in time to be registered separately. Several overlapping pulses are then counted as one hit only at a too high energy ("pulse pile-up"), see Fig. 10. Pulse pile-up leads to non-linear detector count rates and finally to detector saturation ${ }^{4}$. Even though the signal can be linearized before the onset of saturation, significant quantum losses, increased image noise and reduced energy discrimination cannot be avoided. A way out of this dilemma is a reduction of the pixel size of the detector - however, smaller pixels lead to more charge sharing and $\mathrm{K}$ escape. Finding the optimum size of the detector cells to balance pulse pile-up, charge sharing and K-escape is one of the most challenging tasks

\footnotetext{
${ }^{4} \mathrm{Si}$-based photon counting detectors have a higher charge carrier mobility than CdTe/CZT. They can therefore handle higher x-ray fluxes without suffering from pulse pile-up [64].
}

in designing a photon-counting detector.

Another problem of photon-counting detectors is count-rate drift at higher x-ray flux rates. Non-homogeneously distributed crystal defects in the sensor material cause trapping of electrons and holes - the resulting space charges modify the electric field distribution differently in the individual detector pixels. This changes the characteristics of the signal pulses depending on the "irradiation history" of the respective pixels and may lead to severe ring artifacts in the images at higher flux rates.

\section{Pre-clinical evaluation of photon-counting CT}

Photon-counting detectors are a promising new technology fur future medical CT systems. Currently, pre-clinical prototypes are used to evaluate the potential and limitations of photon-counting CT in clinical practice. We focus on these pre-clinical installations and leave out other more experimental solutions, benchtop systems, and photon-counting micro CT systems.

Silicon-based photon counting detectors were first evaluated for dedicated breast CT imaging [65], but the scope was soon extended to other applications. Synthetic monoenergetic, virtual non-contrast and virtual non-calcium images of a heart sample could be obtained with an experimental benchtop CT scanner with 8 energy bins [66]. Meanwhile, a prototype single source CT scanner with a full-field-of-view siliconbased photon-counting detector capable of patient scanning has been presented [67]. The system provides improved spatial resolution of 19 $\mathrm{lp} / \mathrm{cm}$, compared to $14 \mathrm{lp} / \mathrm{cm}$ for a reference scanner [67].

There are several pre-clinical prototype CT-systems equipped with CdTe- or CZT-detectors.

A small-bore spectral micro-CT based on a Medipix-detector with 8 energy channels has been translated to a large-bore photon counting CT capable of obtaining diagnostic spectral CT images of a human within a clinical radiation dose level [68], however, no further results have been published yet.

A pre-clinical single source CT system with photon-counting detector based on CZT (Philips Healthcare, Haifa, Israel) provides an in-plane field of view of $168 \mathrm{~mm}$ and a z-coverage of $2.5 \mathrm{~mm}$, with a rotation time of $1 \mathrm{~s}$ [25]. The size of the detector pixels is $0.5 \times 0.5 \mathrm{~mm}^{2}$. The photon-counting detector has 5 energy thresholds. The system was evaluated both with phantoms and with animal scans, demonstrating improved assessment of lung structures due to higher resolution [25] and improved visualization of the in-stent lumen and in-stent re-stenosis 

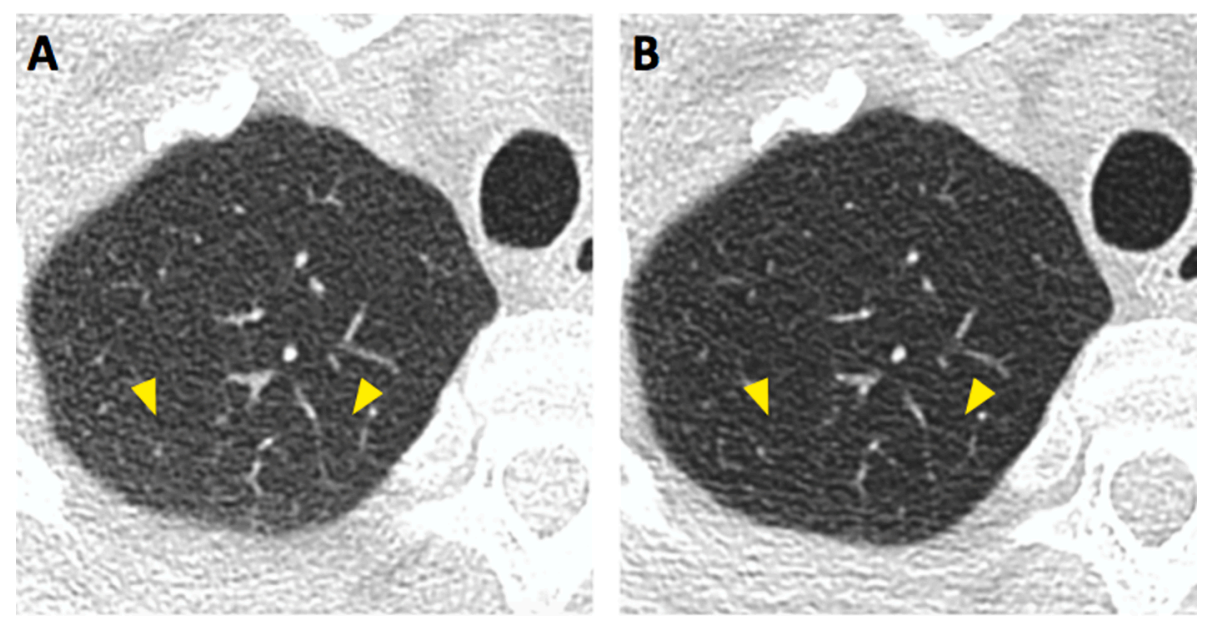

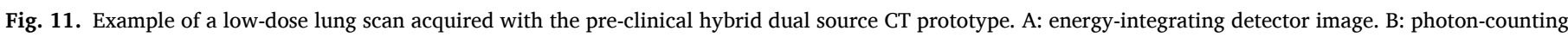

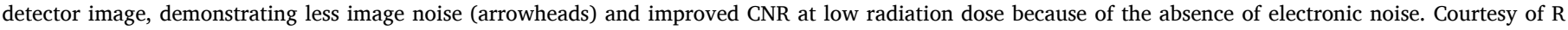
Symons, NIH, Bethesda, MD, USA.

in coronary stents [26]. Differentiation of several contrast agents by multi-material decomposition was demonstrated ([27, 28,29,30,31,32]), with various potential clinical applications. Differentiation between blood and iodine in a bovine brain was demonstrated as well by computing iodine maps and virtual non-contrast images [33].

A pre-clinical hybrid dual source CT scanner is equipped with a conventional scintillation detector and a CdTe photon-counting detector (Siemens Healthcare $\mathrm{GmbH}$, Forchheim, Germany). The photoncounting detector consists of sub-pixels with a size of $0.225 \times 0.225$ $\mathrm{mm}^{2}$. The detector provides 2 energy thresholds per sub-pixel. $2 \times 2$ subpixels can be binned to a "sharp pixel" or "ultra-high resolution (UHR) pixel" with a pixel size of $0.45 \times 0.45 \mathrm{~mm}^{2}, 4 \times 4$ sub-pixels can be binned to a "macro pixel" with a size of $0.9 \times 0.9 \mathrm{~mm}^{2}$ comparable to today's medical CT systems. By assigning alternating low-energy and highenergy thresholds to adjacent detector sub-pixels in a "chess pattern" mode, the detector provides 4 energy thresholds in "macro pixels". The in-plane field-of-view of the photon-counting detector is $275 \mathrm{~mm}$, the zcoverage is $8-16 \mathrm{~mm}$, depending on the read-out mode. A completion scan with the energy-integrating sub-system can be used to extend the photon-counting field of view to $500 \mathrm{~mm}$. The shortest rotation time of the system is $0.5 \mathrm{~s}$. The x-ray tubes (Straton, Siemens Healthcare GmbH, Forchheim, Germany) can be operated at voltages up to $140 \mathrm{kV}$, with a tube current up to $550 \mathrm{~mA}$ (maximum tube power $77 \mathrm{~kW}$ ). The focal spot size is $1.40 \times 1.40 \mathrm{~mm}^{2}$ (width $\times$ length) in the "macro pixel" mode and $1.0 \times 1.0 \mathrm{~mm}^{2}$ in the "sharp" or "UHR" mode, for the operation conditions of the clinical examples presented later. The pre-clinical hybrid dual source CT scanner was described and evaluated in ([34,35,36]).

Yet another pre-clinical single source CT scanner (Siemens Healthcare $\mathrm{GmbH}$, Forchheim, Germany) is equipped with a CdTe photoncounting detector with a similar pixel geometry as the hybrid dual source CT. The photon-counting detector consists of sub-pixels with a size of $0.275 \times 0.322 \mathrm{~mm}^{2}$. Its field-of-view is $500 \mathrm{~mm}$ at the iso-center. $2 \mathrm{x} 2$ sub-pixels can be binned to a "macro pixel" in the "standard" mode, the z-coverage is then $57.6 \mathrm{~mm}(144 \times 0.4 \mathrm{~mm}$ at the iso-center). The sub-pixels can as well be read out separately in the "UHR" mode, the $\mathrm{z}$-coverage is then limited to $24 \mathrm{~mm}(120 \mathrm{x} 0.2 \mathrm{~mm} \text { at the iso-center })^{5}$. The detector provides 4 energy thresholds per sub-pixel. The shortest rotation time of the system is $0.3 \mathrm{~s}$. The system is equipped with an $\mathrm{x}$-ray tube (Vectron, Siemens Healthcare GmbH, Forchheim, Germany) with a focal spot size of $1.00 \times 1.40 \mathrm{~mm}^{2}$ (width $\times$ length) in the "standard" mode (providing up to $120 \mathrm{~kW}$ tube power) and $0.7 \times 0.9 \mathrm{~mm}^{2}$ in the "UHR" mode (providing up to $50 \mathrm{~kW}$ tube power).

The imaging performance of the pre-clinical hybrid dual source CT was evaluated by means of phantom and cadaver scans ([37,38]), confirming clinical image quality at clinically realistic levels of x-ray photon flux. In contrast-enhanced abdominal scans of human volunteers, photon-counting detector images showed similar qualitative and quantitative image quality scores as conventional CT images, while additionally providing spectral information for material decomposition [39].

The improvement of iodine CNR by photon-counting CT, which is expected as a result of the missing down-weighting of low-energy x-ray photons, was confirmed by measurements in 4 anthropomorphic phantoms simulating 4 patient sizes ([38]). A mean increase in iodine CNR of $11 \%, 23 \%, 31 \%, 38 \%$ relative to the scintillation detector system at $80,100,120$, and $140 \mathrm{kV}$, respectively, was shown. These improvements in iodine CNR can potentially be translated into reduced radiation dose, or reduced amount of contrast agent. Improvement of soft-tissue contrasts was demonstrated in a brain CT study with 21 human volunteers [40]. The higher reader scores for the differentiation of grey and white brain matter for photon-counting CT images compared to conventional CT images were attributed to both higher soft-tissue contrasts $(10.3 \pm 1.9 \mathrm{HU}$ versus $8.9 \pm 1.8 \mathrm{HU})$, and lower image noise for photoncounting CT.

The impact of missing electronic noise on image quality was assessed for various clinical applications at low radiation dose. Less streaking artifacts in shoulder images acquired with the photon-counting detector of the pre-clinical hybrid dual source CT as compared to its scintillation detector were demonstrated [41]. Symons et al [42] found better Hounsfield unit stability for lung, ground-glass, and emphysemaequivalent foams of a lung phantom in combination with better

\footnotetext{
5 The effective size of a sub-pixel is somewhat larger because of additional dead-zones due to collimator blades after every 6th sub-pixel in the z-direction and after every 4th sub-pixel in the phi-direction, see also Fig. 3 for an illustration. With an effective sub-pixel size of $1.13 \times 0.322 \mathrm{~mm}$ in the z-direction, a focus-isocenter distance of $610 \mathrm{~mm}$ and a focus-detector distance of $1113 \mathrm{~mm}$ the "slice width" of a sub-pixel at the isocenter is $610 / 1113 * 1.13 * 0.322 \mathrm{~mm}=$ $0.20 \mathrm{~mm}$.
} 

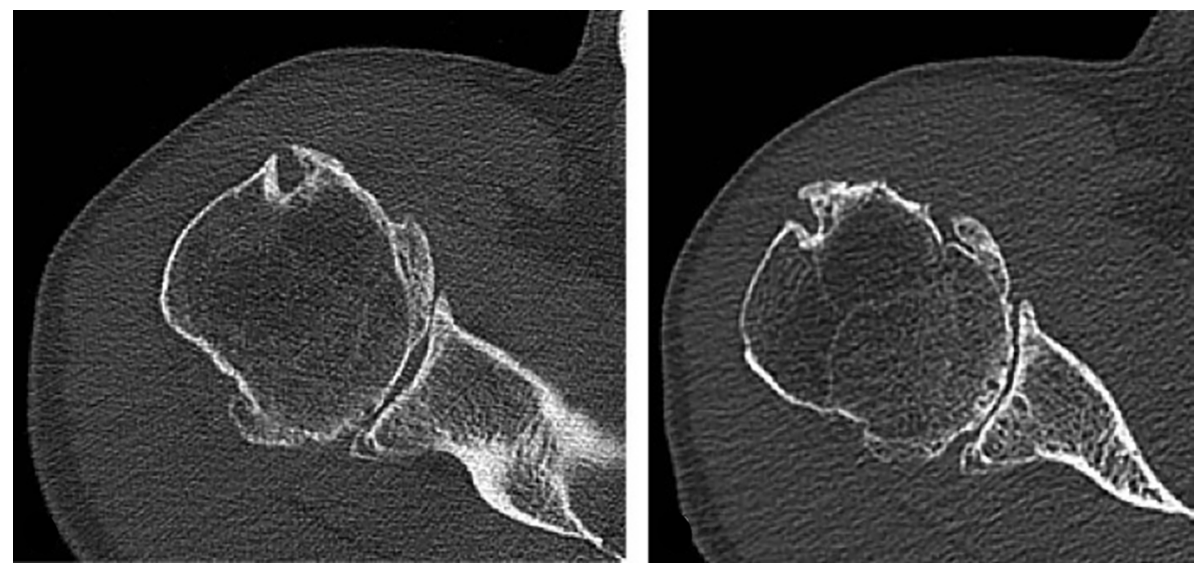

Fig. 12. Example of a shoulder scan acquired with the pre-clinical hybrid dual source CT prototype. Left: energy-integrating detector image. Right: photon-counting detector image, "sharp" mode, demonstrating higher spatial resolution and significantly improved visualization of bony structures. Modified from [45].

reproducibility of the measurements. This is an important pre-requisite for further reduced radiation dose in lung imaging, e. g. in the context of lung cancer screening. In a study with 30 human subjects undergoing dose-reduced chest CT imaging [43], photon-counting CT demonstrated higher diagnostic quality with significantly better image quality scores for lung, soft tissue, and bone, fewer beam-hardening artifacts, lower image noise, and higher CNR for lung nodule detection, see Fig. 11.

Improved quality of coronary artery calcium (CAC) scoring at low radiation dose was shown in a combined phantom, ex-vivo and in-vivo study [44]. Agreement between standard-dose (average CTDI $_{\mathrm{vol}}=5.4$ $\mathrm{mGy}$ ) and low-dose (average CTDI $\mathrm{vol}_{\mathrm{v}}=1.6 \mathrm{mGy}$ ) CAC score in 10 volunteers was significantly better for photon-counting CT than for conventional CT, attributed to the absence of electronic noise in combination with improved calcium-soft tissue contrasts due to missing down-weighting of low-energy x-ray photons. The authors concluded that photon-counting CT technology may play a role in further reducing the radiation dose of CAC scoring.

Improvements in spatial resolution with the pre-clinical hybrid dual source CT enabled by the smaller pixels of its photon-counting detector in "sharp" mode and in "UHR" mode were evaluated in several phantom studies. $150 \mu \mathrm{m}$ in-plane spatial resolution and minimum slice widths down to $0.41 \mathrm{~mm}$ were demonstrated, and better spatial resolution was confirmed in clinical images of the lung, shoulder and temporal bone [45], see Fig. 12. At equal spatial resolution, photon-counting images had less image noise than conventional CT images because of the better modulation transfer function (MTF) of the measurement system. Significant improvements of coronary stent lumen visibility with the "UHR" mode were found [46], as well as superior qualitative and quantitative image characteristics for coronary stent imaging when using a dedicated sharp convolution kernel [47].

In a small study with 8 humans undergoing scans of the brain, the thorax, and the left kidney, Pourmorteza et al [48] observed improved spatial resolution and less image noise with the "UHR" mode compared with standard-resolution photon-counting CT ("macro" mode). Substantially better delineation of temporal bone anatomy scanned with the "UHR" mode compared with the ultra-high-resolution mode of a commercial energy-integrating-detector CT scanner was shown in [49].

Superior visualization of higher-order bronchi and third-/fourthorder bronchial walls at preserved lung nodule conspicuity compared with clinical reference images was demonstrated in 22 adult patients referred for clinically indicated high-resolution chest CT [50]. The authors combined "sharp" modephoton-counting CT with image

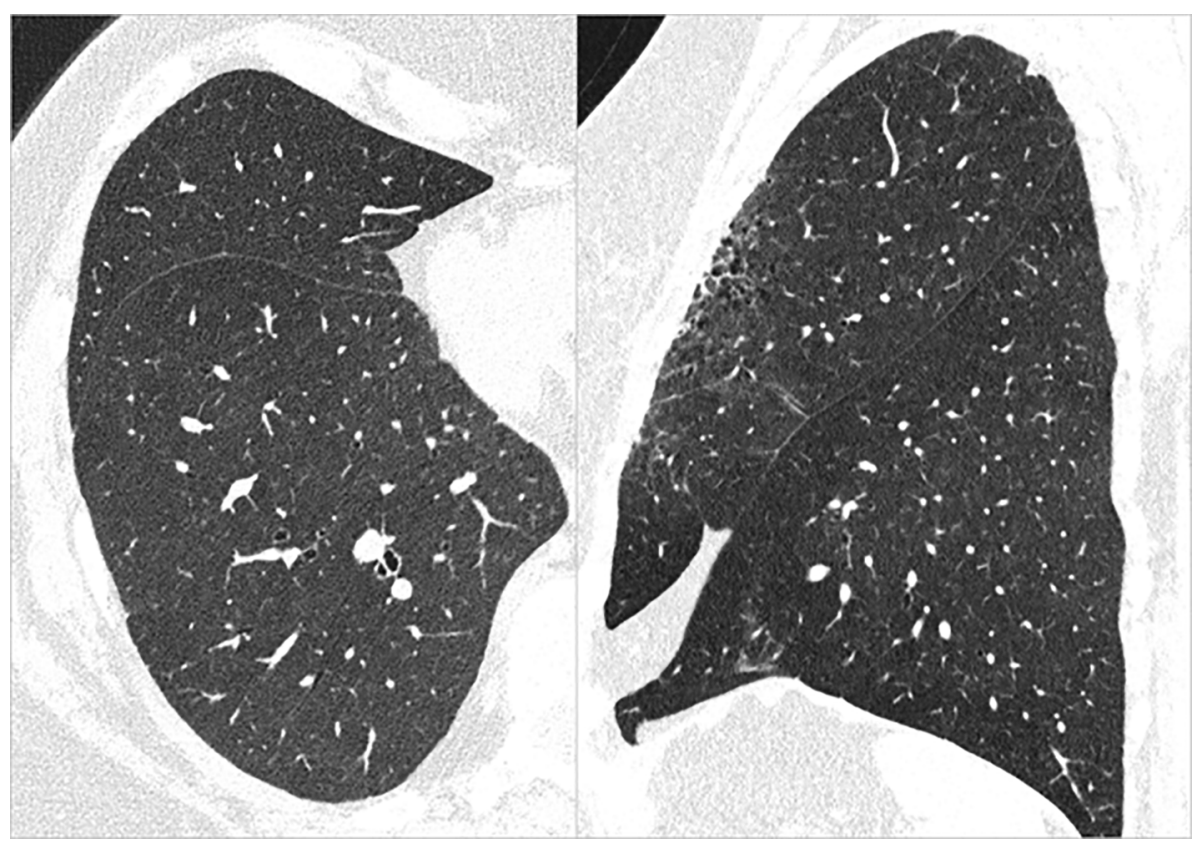

Fig. 13. Lung images of a 74-year old woman with breast cancer and signs of fibrosis after radiation therapy, acquired with a pre-clinical single source CT prototype with photon-counting detector. Data acquisition: "UHR" mode, $120 \times 0.2 \mathrm{~mm}$ collimation, 0.3 s rotation time, CTDI $_{\mathrm{vol}}=3.89 \mathrm{mGy}, \mathrm{DLP}=126$ mGycm. Image reconstruction: sharp convolution kernel, 1024x1024 image matrix, $0.4 \mathrm{~mm}$ slice width. Excellent visualization of fibrosis and fine details such as fissures. Courtesy of Dr. J. Ferda, Pilsen, Czech Republic. 


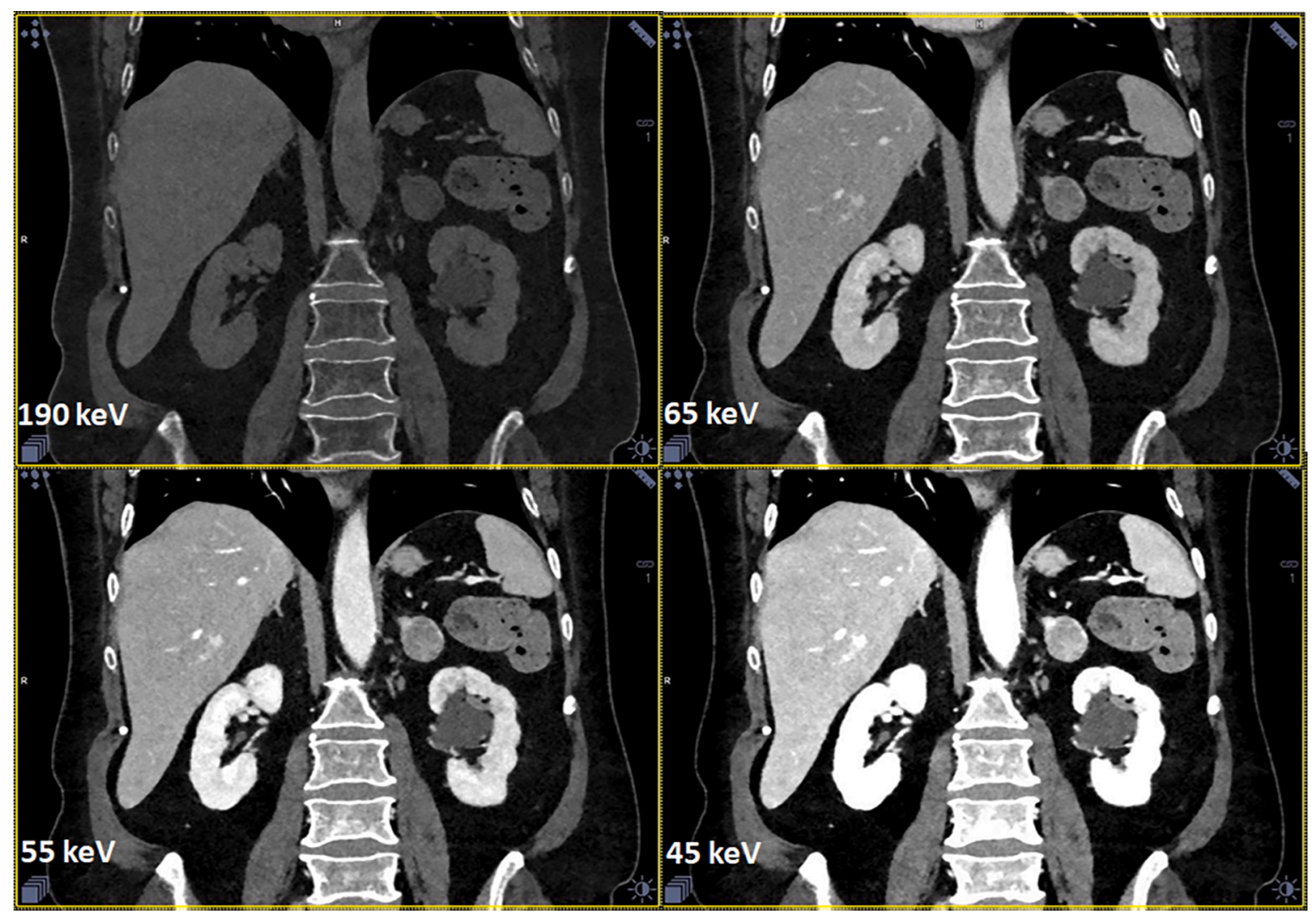

Fig. 14. Abdominal images of a 67 -year old woman with adrenal adenoma and parapelvic renal cyst, acquired with a pre-clinical single source CT prototype with photon-counting detector. Data acquisition: "standard" mode, $144 \mathrm{x} 0.4 \mathrm{~mm}$ collimation, $0.3 \mathrm{~s}$ rotation time, CTDI $\mathrm{vol}=10.2 \mathrm{mGy}$, DLP $=450 \mathrm{mGycm}$. Image reconstruction: VMIs at $190 \mathrm{keV}, 65 \mathrm{keV}, 55 \mathrm{keV}$ and $45 \mathrm{keV}, 0.4 \mathrm{~mm}$ slice width. Note the decreasing contrast of iodine and calcium with increasing keV - the 190 $\mathrm{keV}$ image can serve as a substitute for a virtual non-contrast image. Courtesy of Dr. J. Ferda, Pilsen, Czech Republic.

reconstruction at 1024 x1024 matrix size using a dedicated sharp convolution kernel. According to the authors, photon-counting CT is beneficial for high-resolution imaging of airway diseases, and potentially for other pathologies, such as fibrosis, honeycombing, and emphysema.

The achievable image quality with a photon-counting detector in high-resolution chest CT is demonstrated in Fig. 13.

Practically, spatial resolution does not only depend on the detector pixel size, but also on the focal spot size of the x-ray tube which needs to be correspondingly small. The smaller the focal spot is, the less tube power is usually available, which may limit the clinical applicability of ultra-high-resolution CT scanning. Furthermore, increased resolution comes at the expense of increased image noise if the radiation dose is kept constant. Increased radiation dose to the patient to compensate for the higher noise may not be acceptable in all cases. Non-linear data and image denoising techniques will therefore play a key role in harnessing the high-resolution potential of photon-counting detectors, see e. g. $([51,52,53])$

A key benefit of photon-counting CT is spectrally resolved data acquisition in any scan.

The spectral performance of the pre-clinical hybrid dual source prototype with photon-counting detector was evaluated in phantom studies [54], and the CT number accuracy in VMIs and iodine quantification accuracy were found to be comparable to dual source dualenergy CT. According to the authors, photon-counting CT offers additional advantages, such as perfect temporal and spatial alignment to avoid motion artifacts, high spatial resolution, and improved CNR. In an anthropomorphic head phantom containing tubes filled with aqueous solutions of iodine $(0.1-50 \mathrm{mg} / \mathrm{ml})$ excellent agreement between actual iodine concentrations and iodine concentrations measured in the iodine maps was observed [55]. The authors assessed the use of iodine maps and VMIs in head and neck CTA in 16 asymptomatic volunteers and proposed VMIs as a method to enhance plaque detection and characterization as well as grading of stenosis by reconstructing images at different keV.

The routine availability of VMIs with photon-counting CT may pave the way to further standardization of CT-protocols, provided that CNR and image quality of the VMIs are enhanced by refined processing (see e. g. [56]). In this approach, VMIs at standardized keV levels tailored to the clinical question (e. g. 50-70 keV for contrast-enhanced examinations of parenchymal organs, $40-50 \mathrm{keV}$ for CT angiographic studies) are the primary output of any CT scan regardless of the acquisition protocol, see Fig. 14. Going one step further, the acquisition protocol may be standardized as well. Some authors [57] already recommend a standardized acquisition protocol with $140 \mathrm{kV}$ x-ray tube voltage for contrastenhanced abdominal CT examinations in all patient sizes, with standardized VMI reconstruction at $50 \mathrm{keV}$. According to the authors, optimal or near optimal iodine CNR for all patient sizes is obtained with this protocol.

Several authors assessed the performance of spectral photoncounting CT for detection and characterization of kidney stones, another established dual-energy CT application ([58,59,60]). They found comparable overall performance to state-of-the art dual-energy CT in differentiating stone composition, while photon-counting CT was better able to help characterize small renal stones ([60]).

If the photon-counting detector is operated with more than two energy bins, multi-material decomposition is possible if K-edge elements are present. In a canine model of myocardial infarction, Symons et al [61] performed dual-contrast agent imaging of the heart to simultaneously assess both first-pass and late enhancement of the myocardium. The authors concluded that combined first-pass iodine and late gadolinium maps allowed quantitative separation of blood pool, infarct scar, 

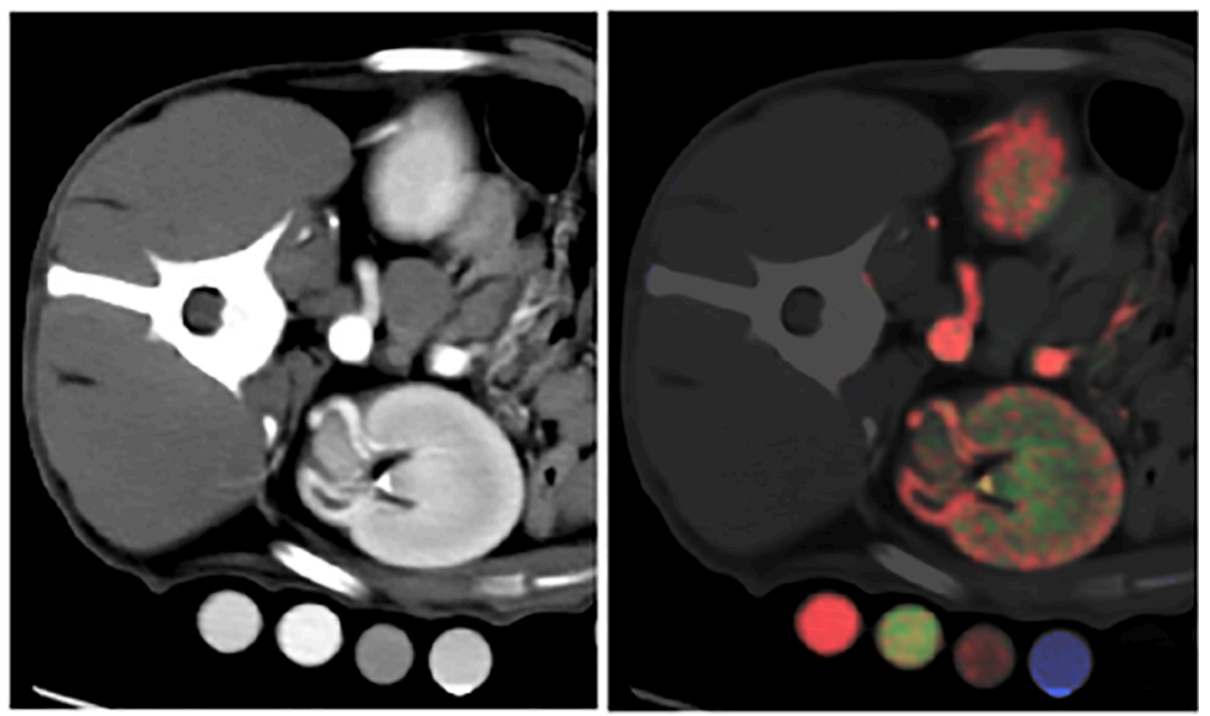

Fig. 15. Simultaneous imaging of 3 different contrast agents (iodine, gadolinium and bismuth) by multi-material decomposition in a dog model. Scan data were acquired with the pre-clinical hybrid dual source CT prototype. Bismuth was administered more than one day prior to scanning, followed by intravenous administration of gadolinium-based contrast agent 3-5 min before the scan and iodinebased contrast agent immediately before the scan to visualize different phases of renal enhancement in a single CT acquisition. Scan data were read-out in four energy bins (25-50 keV, 50-75 keV, 75-90 keV, and $90-140 \mathrm{keV})$. Left: grayscale image using all detected $x$-ray photons by combining the data of the four energy bins. The three contrast agents cannot be differentiated. Right: grayscale-image with overlay of the colored material maps. Iodine is color-coded in red, gadolinium in green, bismuth in blue. Modified from [62]. (For interpretation of the references to color in this figure legend, the reader is referred to the web version of this article.)

and remote myocardium. The same authors also investigated the feasibility of simultaneous material decomposition of three contrast agents (bismuth, iodine and gadolinium) in vivo in a canine model [62]. They observed tissue enhancement at multiple phases in a single CT acquisition, opening the potential to replace multiphase CT scans by a single CT acquisition with multiple contrast agents, see Fig. 15.

In clinical practice, the use of multi-material maps may be hampered by the unavoidable increase of image noise in a multi-material decomposition. Similar to ultra-high resolution scanning non-linear data and image denoising techniques will play a key role to fully exploit the potential of multi-material decomposition in clinical routine, see e. g. [63]

In this review article, we have outlined the basic principles of photon-counting CT and its potential clinical applications. Once remaining challenges of this technology have been mastered, photoncounting CT has the potential to bring clinical CT to a new level of performance.

\section{References}

[1] Kalender W, Seissler W, Klotz E, Vock P. Spiral volumetric CT with single-breathhold technique, continuous transport and continuous scanner rotation. Radiology 1990;176:181-3.

[2] McCollough CH, Zink FE. Performance Evaluation of a Multi-slice CT System. Med Phys 1999;26:2223-30.

[3] Klingenbeck-Regn K, Schaller S, Flohr T, Ohnesorge B, Kopp AF, Baum U. Subsecond multi-slice computed tomography: basics and applications. Eur J Radiol 1999;31:110-24.

[4] Mori S, Obata T, Nakajima N, Ichihara N, Endo M. Volumetric perfusion CT using prototype 256-detector row CT scanner: preliminary study with healthy porcine model. AJNR Am J Neuroradiol 2005;26(10):2536-41.

[5] Flohr TG, McCollough CH, Bruder H, Petersilka M, Gruber K, Süß C, et al. First performance evaluation of a dual-source CT (DSCT) system. Eur Radiol 2006;16: $256-68$.

[6] Vanhecke TE, Madder RD, Weber JE, et al. Development and validation of a predictive screening tool for uninterpretable coronary CT angiography results. Circ Cardiovasc Imaging. 2011;4(5):490-7.

[7] Shanbhag SM, Schuzer JL, Steveson C, Rollison S, Bronson KC, Stagliano MS, et al Prototype Ultrahigh-Resolution Computed Tomography for Chest Imaging: Initial Human Experience. J Comput Assist Tomogr 2019;43(5):805-10.

[8] Duan X, Wang J, Leng S, et al. Electronic noise in CT detectors: Impact on image noise and artifacts. AJR Am J Roentgenol 2013 Oct;201(4):W626-32.

[9] Lu GM, Zhao Y, Zhang LJ, Schoepf UJ. Dual-energy CT of the lung. AJR Am J Roentgenol 2012;199(5 Suppl):S40-53.

[10] Marin D, Boll DT, Mileto A, Nelson RC. State of the art: dual-energy CT of the abdomen. Radiology 2014;271(2):327-42.

[11] Odisio EG, Truong MT, Duran C, de Groot PM, Godoy MC. Role of Dual-Energy Computed Tomography in Thoracic Oncology. Radiol Clin North Am 2018;56(4): 535-48.

[12] Albrecht MH, De Cecco CN, Schoepf UJ, et al. Dual-energy CT of the heart current and future status. Eur J Radiol 2018;105:110-8.
[13] De Santis D, Eid M, De Cecco CN, et al. Dual-Energy Computed Tomography in Cardiothoracic Vascular Imaging. Radiol Clin North Am 2018;56(4):521-34.

[14] Rajiah P, Sundaram M, Subhas N. Dual-Energy CT in Musculoskeletal Imaging: What Is the Role Beyond Gout? AJR Am J Roentgenol 2019;213(3):493-505.

[15] Siegel MJ, Ramirez-Giraldo JC. Dual-Energy CT in Children: Imaging Algorithms and Clinical Applications. Radiology 2019;291(2):286-97.

[16] Johnson TRC, Krauß B, Sedlmair M, et al. (2007). Material differentiation by dualenergy CT: initial experience. Eur Radiol 2007; 17(6):1510-7.

[17] Zhang D, Li X, Liu B. Objective characterization of GE discovery CT750 HD scanner: gemstone spectral imaging mode. Med Phys 2011;38(3):1178-88.

[18] Rassouli N, Etesami M, Dhanantwari A, Rajiah P. Detector-based spectral CT with a novel dual-layer technology: principles and applications. Insights Imaging 2017;8 (6):589-98.

[19] Feuerlein S, Roessl E, Proksa R, et al. Multienergy photon-counting K-edge imaging: potential for improved luminal depiction in vascular imaging. Radiology 2008;249(3):1010-6.

[20] Taguchi K, Iwanczyk JS. Vision 20/20: Single photon-counting x-ray detectors in medical imaging. Med Phys 2013;40(10):100901.

[21] Taguchi K. Energy-sensitive photon-counting detector-based X-ray computed tomography. Radiol Phys Technol 2017;10(1):8-22.

[22] Willemink MJ, Persson M, Pourmorteza A, Pelc NJ, Fleischmann D. Photoncounting CT: Technical Principles and Clinical Prospects. Radiology 2018;289(2): 293-312.

[23] Leng S, Bruesewitz M, Tao S, et al. Photon-counting Detector CT: System Design and Clinical Applications of an Emerging Technology. Radiographics 2019;39(3): $729-43$.

[24] Kappler S, Niederlöhner D, Stierstorfer K, Flohr T. Contrast-enhancement, image noise and dual-energy simulations for quantum-counting clinical CT. Proceedings of the SPIE Medical Imaging Conference 2010;7622:76223H.

[25] Kopp FA, Daerr H, Si-Mohamed S, et al. Evaluation of a pre-clinical photoncounting CT prototype for pulmonary imaging. Sci Rep 2018;8(1):17386.

[26] Bratke G, Hickethier T, Bar-Ness D, et al. Spectral Photon-Counting Computed Tomography for Coronary Stent Imaging: Evaluation of the Potential Clinical Impact for the Delineation of In-Stent Restenosis. Invest Radiol 2019 Sep 12. doi: 10.1097/RLI.0000000000000610. [Epub ahead of print].

[27] Riederer I, Bar-Ness D, Kimm MA. Liquid embolics agents in spectral x-ray photoncounting computed tomography using tantalum K-edge imaging. Sci Rep 2019;9: 5268.

[28] Cormode DP, Si-Mohamed S, Bar-Ness D, et al. Multicolor spectral photon-counting computed tomography: in vivo dual contrast imaging with a high count rate scanner. Sci Rep 2017;7(1):4784.

[29] Dangelmaier J, Bar-Ness D, Daerr H, et al. Experimental feasibility of spectral photon-counting computed tomography with two contrast agents for the detection of endoleaks following endovascular aortic repair. Eur Radiol 2018;28(8):3318-25.

[30] Muenzel D, Bar-Ness D, Roessl E, et al. Spectral Photon-counting CT: Initial Experience with Dual-Contrast Agent K-Edge Colonography. Radiology 2017;283 (3):723-8.

[31] Si-Mohamed S, Bar-Ness D, Sigovan M, et al. Multicolour imaging with spectral photon-counting CT: a phantom study. Eur Rad Experimental 2018;2:34.

[32] Si-Mohamed S, Tatard-Leitman V, Laugerette A, et al. Spectral Photon-Counting Computed Tomography (SPCCT): in-vivo single-acquisition multi-phase liver imaging with a dual contrast agent protocol. Sci Rep 2019;9(1):8458.

[33] Riederer I, Si-Mohamed S, Ehn S, et al. Differentiation between blood and iodine in a bovine brain - Initial experience with spectral photon-counting computed tomography (SPCCT). PLoS ONE 2019;14(2):e0212679.

[34] Kappler S, Hannemann T, Kraft E, et al. First results from a hybrid prototype CT scanner for exploring benefits of quantum-counting in clinical CT. Medical Imaging 
2012: Physics of Medical Imaging (San Diego, CA: International Society for Optics and Photonics) p 83130X.

[35] Kappler S, Henning A, Krauss B, et al. Multi-energy performance of a research prototype CT scanner with small-pixel counting detector. Medical Imaging 2013: Physics of Medical Imaging (Lake Buena Vista, FL: International Society for Optics and Photonics) p. 866800.

[36] Kappler S, Henning A, Kreisler B, et al. Photon-counting CT at elevated x-ray tube currents: contrast stability, image noise and multi-energy performance. Medical Imaging 2014: Physics of Medical Imaging (San Diego, CA: International Society for Optics and Photonics) p. 90331C.

[37] Yu Z, Leng S, Jorgensen SM, et al. Evaluation of conventional imaging performance in a research CT system with a photon-counting detector array. Phys Med Biol 2016;61:1572-95.

[38] Gutjahr R, Halaweish AF, Yu Z, et al. Human Imaging With Photon-counting-Based Computed Tomography at Clinical Dose Levels: Contrast-to-Noise Ratio and Cadaver Studies. Invest Radiol 2016;51(7):421-9.

[39] Pourmorteza A, Symons R, Sandfort V, et al. Abdominal Imaging with contrastenhanced photon-counting CT: first human experience. Radiology 2016;279(1): $239-45$.

[40] Pourmorteza A, Symons R, Reich DS, Bagheri M, Cork TE, Kappler S, et al. PhotonCounting CT of the Brain. In Vivo Human Results and Image-Quality Assessment. AJNR Am J Neuroradiol 2017;38(12):2257-63.

[41] Yu Z, Leng S, Kappler S, et al. Noise performance of low-dose CT_ comparison between an energy integrating detector and a photon-counting detector using a whole-body research photon-counting CT scanner. J Med Imaging 2016;3(4): 043503.

[42] Symons R, Cork T, Sahbaee P, et al. Low-dose lung cancer screening with photoncounting CT: a feasibility study. Phys Med Biol 2017;62(1):202-13.

[43] Symons R, Pourmorteza A, Sandfort V, et al. Feasibility of Dose-reduced Chest CT with Photon-counting Detectors: Initial Results in Humans. Radiology 2017;285 (3):980-9.

[44] Symons R, Sandfort V, Mallek M, Ulzheimer S, Pourmorteza A. Coronary artery calcium scoring with photon-counting CT: first in vivo human experience. Int $\mathrm{J}$ Cardiovasc Imaging 2019;35(4):733-9.

[45] Leng S, Rajendran K, Gong H, et al. 150- $\mu \mathrm{m}$ Spatial Resolution Using PhotonCounting Detector Computed Tomography Technology: Technical Performance and First Patient Images. Invest Radiol 2018;53(11):655-62.

[46] Symons R, de Bruecker Y, Roosen J, et al. Quarter-millimeter spectral coronary stent imaging with photon-counting CT: Initial experience. J Cardiovascul Comp Tomogr 2018;12:509-15.

[47] von Spiczak J, Mannil M, Peters B, et al. Photon-counting computed Tomography with dedicated sharp convolution kernels - tapping the potential of a new technology for stent imaging. Invest Radiol 2018;53(8):486-94.

[48] Pourmorteza A, Symons R, Henning A, Ulzheimer S, Bluemke DA. Dose Efficiency of Quarter-Millimeter Photon-Counting Computed Tomography: First-in-Human Results. Invest Radiol 2018;53(6):365-72.

[49] Zhou W, Lane JI, Carlson ML, et al. Comparison of a Photon-Counting-Detector CT with an Energy-Integrating-Detector CT for Temporal Bone Imaging: A Cadaveric Study. AJNR Am J Neuroradiol 2018;39(9):1733-8.

[50] Bartlett DJ, Koo WC, Bartholmai BJ, et al. High-Resolution Chest Computed Tomography Imaging of the Lungs: Impact of 1024 Matrix Reconstruction and Photon-Counting Detector Computed Tomography. Invest Radiol 2019;54(3): 129-37.

[51] Li Z, Leng S, Yu L, et al. An effective noise reduction method for multi-energy CT images that exploit spatio-spectral features. Med Phys 2017;44(5):1610-23.

[52] Harrison AP, Xu Z, Pourmorteza A, Bluemke DA, Mollura DJ. A multichannel block-matching denoising algorithm for spectral photon-counting CT images. Med Phys 2017;44(6):2447-52.

[53] Rajendran K, Tao S, Abdurakhimova D, Leng S, McCollough C. Ultra-high resolution photon-counting detector CT reconstruction using spectral prior image constrained compressed-sensing (UHR-SPICCS). Proc SPIE Int Soc Opt Eng; 2018 Mar;10573. pii: 1057318. doi: 10.1117/12.2294628.
[54] Leng S1, Zhou W, Yu Z, et al. Spectral performance of a whole-body research photon-counting detector CT: quantitative accuracy in derived image sets. Phys Med Biol 2017;62(17):7216-32.

[55] Symons R, Reich DS, Bagheri M, et al. Photon-Counting Computed Tomography for Vascular Imaging of the Head and Neck: First In Vivo Human Results. Invest Radiol 2018;53(3):135-42.

[56] Grant KL, Flohr TG, Krauss B, et al. Assessment of an Advanced Image-Based Technique to Calculate Virtual Monoenergetic Computed Tomographic Images From a Dual-Energy Examination to Improve Contrast-To-Noise Ratio in Examinations Using Iodinated Contrast Media. Invest Radiol 2014;49(9):586-92.

[57] Zhou W, Abdurakhimova D, Bruesewitz M, et al. Impact of photon-counting detector technology on $\mathrm{kV}$ selection and diagnostic workflow in CT. Proc SPIE Int Soc Opt Eng. 201 Mar;10573. pii: 105731C doi: 10.1117/12.2294952.

[58] Gutjahr R, Polster C, Henning A, Kappler S, Leng S, McCollough CH, et al. Dualenergy CT Kidney Stone Differentiation in Photon-counting Computed Tomography. Proc SPIE Int Soc Opt Eng 2017;10132.

[59] Ferrero A, Gutjahr R, Halaweish AF, Leng S, McCollough CH. Characterization of Urinary Stone Composition by Use of Whole-body, Photon-counting Detector CT. Acad Radiol 2018;25(10):1270-6.

[60] Marcus RP, Fletcher JG, Ferrero A, et al. Detection and Characterization of Renal Stones by Using Photon-Counting-based CT. Radiology 2018;289(2):436-42.

[61] Symons R, Cork TE, Lakshmanan MN, et al. Dual-contrast agent photon-counting computed tomography of the heart: initial experience. Int J Cardiovasc Imaging 2017;33:1253-61.

[62] Symons R, Krauss B, Sahbaee P, et al. Photon-counting CT for simultaneous imaging of multiple contrast agents in the abdomen: An in vivo study. Med Phys 2017;44(10):5120-7.

[63] Tao S, Rajendran K, McCollough CH, Leng S. Material decomposition with prior knowledge aware iterative denoising (MD-PKAID). Phys Med Biol 2018;63(19): 195003.

[64] Persson M, Huber B, Karlsson S, et al. Energy-resolved CT imaging with a photoncounting silicon-strip detector. Phys Med Biol 2014;59:6709-27.

[65] Cho HM, Barber WC, Ding H, Iwanczyk JS, Molloi S. Characteristic performance evaluation of a photon counting Si strip detector for low dose spectral breast CT imaging. Med Phys 2014;41(9):091903.

[66] Grönberg F, Lundberg J, Sjölin M, et al. Feasibility of unconstrained three-material decomposition: imaging an excised human heart using a prototype silicon photoncounting CT detector. Eur Radiol 2020 Jun 25. doi: 10.1007/s00330-020-07017-y. Online ahead of print.

[67] da Silva J, Grönberg F, Cederström B, et al. Resolution characterization of a siliconbased, photon-counting computed tomography prototype capable of patient scanning. J Med Imaging (Bellingham). 2019;6(4):043502.

[68] Panta, RK, Butler APH, de Ruiter NJA, et al. First human imaging with MARS photon-counting CT. 2018 IEEE Nuclear Science Symposium and Medical Imaging Conference Proceedings (NSS/MIC).

[69] Flohr TG, Stierstorfer K, Süß C, et al. Novel ultrahigh resolution data acquisition and image reconstruction for multi- detector row CT. Med Phys 2007;34(5): 1712-23.

[70] Kappler S, Niederlöhner D, Wirth S, Stierstorfer K. A full-system simulation chain for computed tomography scanners. Orlando, FL: IEEE; 2009. p. 3433-6. https:// doi.org/10.1109/NSSMIC.2009.5401779.

[71] Taguchi K, Polster C, Lee O, Stierstorfer K, Kappler S. Spatio-energetic cross talk in photon counting detectors: Detector model and correlated Poisson data generator. Med Phys 2016;43(12):6386-404.

[72] Cammin J, Kappler S, Weidinger T, Taguchi K. Evaluation of models of spectral distortions in photon-counting detectors for computed tomography. J Med Imaging 2016;3(2):023503.

[73] Stierstorfer K. Modeling the frequency-dependent detective quantum efficiency of photon-counting x-ray detectors. Med Phys 2018;45:156-66.

[74] Stierstorfer K, Hupfer M, Köster N. Modeling the DQE(f) of photon-counting detectors: impact of the pixel sensitivity profile. Phys Med Biol 2019;64:105008. 